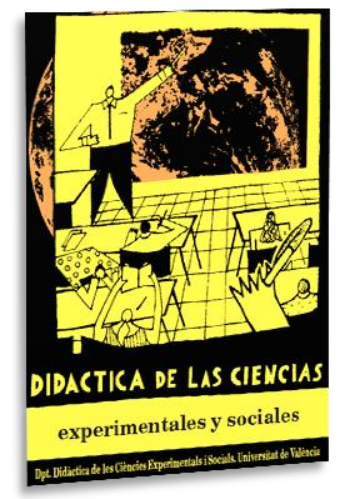

\title{
Del discurso tradicional al modelo innovador en enseñanza de las ciencias: obstáculos para el cambio
}

\author{
From traditional discourse to the innovative \\ model in science education: obstacles to \\ change
}

DOI: 10.7203/DCES.35.12193

\author{
Gabriela Delord \\ Universidad de Sevilla, gabiccd@ hotmail.com \\ ORCID iD: http://orcid.org/0000-0003-2283-5976 \\ Rafael Porlán \\ Universidad de Sevilla, rporlan@us.es \\ ORCID iD: http://orcid.org/0000-0003-2068-7092
}

\begin{abstract}
RESUMEN: En este trabajo se analiza el discurso tradicional de la escuela frente al discurso alternativo del Proyecto IRES (Investigación y Renovación de la Escuela), a través de tres docentes que desarrollan una unidad didáctica de Ciencias. El marco teórico y metodológico utilizado se basa en el Análisis del Discurso. El estudio muestra que incluso en una práctica innovadora y fundamentada aparecen obstáculos que hacen difícil el cambio. Se destacan tres obstáculos relacionados con la autonomía profesional: la dependencia de la dirección del centro, el condicionamiento relativo a la seguridad laboral y la limitación producida por el marco normativo sobre la evaluación. Esta investigación apunta la necesidad de considerar la ideología subyacente en la escuela y su influencia sobre las prácticas innovadoras.
\end{abstract}

Palabras Clave: escuela tradicional, innovación, obstáculos ideológicos, análisis del discurso, enseñanza de las ciencias.

ABSTRACT: In this paper, the traditional discourse in science classes is analyzed against the alternative discourse of the IRES Project (Research and Renewal of the School), through three teachers who develop a didactic unit of Sciences. The theoretical and methodological framework used is based on the Discourse Analysis. The study shows that, even in an innovative and well-founded practice, ideological obstacles appear that make difficult introducing changes. Three obstacles related to professional autonomy stand out: the dependence of the center's management, the conditioning on labor security and the limitation produced by the legislative framework, especially in the evaluation. This research points out the need to consider the underlying ideology in the school and its influence on innovative practices.

KEYWORDS: traditional school, innovation, ideological obstacles, discourse analysis, science education.

Fecha de recepción: marzo de 2018

Fecha de aceptación: abril de 2018 


\section{INTRODUCCIÓN}

La alfabetización científica es considerada una estrategia formativa imprescindible en las sociedades contemporáneas (Garrido y Couso 2013; Gil, 2007), ya que buena parte de los problemas actuales requieren cierto grado de conocimiento crítico de y sobre la ciencia. Al mismo tiempo, la comunidad investigadora estudia más cómo formar profesorado de ciencias innovador para formar alumnos más críticos (Ennis, 2011; Streck, 2009). Sin embargo, la enseñanza de las ciencias no está contribuyendo decididamente al proceso de alfabetización, pues predomina aún una enseñanza transmisiva y conservadora incapaz de promover una ciudadanía que analice y participe en los problemas locales y generales del mundo (Delors, 2003; Menezes, 2000).

Una de las razones de este desfase se relaciona con los obstáculos que tienen los docentes cuando tratan de cambiar sus prácticas. Se han señalado, entre otros, la falta de experiencia en hacer investigación científica (Fisher, Borowski y Tepmer, 2012; Wolffenbuttel, Harres y Delord, 2013), la inadecuada comprensión de la naturaleza de la ciencia (Lederman, Lederman y Antink, 2013), las limitaciones relativas a los contenidos (Duit y Treagust, 2003; Porlán, 1994; Watts y Jofili, 1998), la falta de tiempo e incentivos (Harres, Wolffenbuttel y Delord, 2013) y la diversidad de enfoques del concepto de innovación (Carbonell, 2002; Fullan, 2001; Garcia, 2010).

Desde nuestro punto de vista, es necesario abordar también los obstáculos que el discurso de la escuela tradicional impone a los procesos de cambio. En este sentido, este trabajo se inserta en un estudio más amplio que trata de analizar cómo actúan este tipo de obstáculos en la práctica de docentes comprometidos con el Proyecto IRES (Grupo Investigación en la Escuela, 1991), proyecto que ha sido descrito y analizado anteriormente en Delord, Porlán y Harres (2017) y Delord (2017).

\section{MARCO TEÓRICO}

Althusser (1989) describe la escuela como un aparato ideológico que sirve a los intereses de los sectores dominantes, de ahí que tienda a formar sujetos pasivos y poco discursivos. Otros autores, de acuerdo con esta idea, afirman que la escuela es deudora de una ideología elitista, al servicio de una clase minoritaria interesada en mantener bajos niveles de conciencia crítica en los ciudadanos (Álvarez-Uría, 2002; Hamilton, 2002; Merchán, 2012; Nogueira, 2009). Concretamente, Nogueira (2009) argumenta que, desde el punto de vista de la relación entre el saber y el poder, la escuela ha adquirido el estatus de institución principal al servicio del modelo social neoliberal. Por su parte Álvarez-Uría (2002) afirma irónicamente que la escuela educa para reproducir buenos sujetos, es decir, ciudadanos poco críticos y amoldables que constituyan una sociedad subordinada a los estereotipos sociales.

Siguiendo a estos autores, entendemos que, más allá de los fines declarados, la escuela es un instrumento para la reproducción ideológica, donde su estructura y su dinámica determinan el modelo social que se quiere mantener y reproducir (Torres, 1991), favoreciendo en los sujetos el aprendizaje de la sumisión ante un discurso autoritario, lo que guarda relación, como veremos después, con las dificultades de generalización de los proyectos consecuentemente innovadores y alternativos. Este abordaje teórico, sobradamente conocido, es frecuentemente olvidado en el ámbito de investigación de la enseñanza de las ciencias y debería ser tomado en cuenta en el desarrollo de nuevas propuestas curriculares (Fiala, 2007) y en la formación docente.

Esta perspectiva nos ha llevado a tomar el Análisis del Discurso (AD) como marco teórico y metodológico para la investigación. El AD tiene una importante aceptación en las Ciencias Sociales pues analiza los efectos que tienen los discursos sobre las realidades sociales, ya que se consideran constituidos por signos de naturaleza no sólo lingüística, sino también ideológica (Santander, 2011). Para Ibáñez (2003), la dicotomía mente/mundo es reemplazada por la dualidad discurso/mundo. El lenguaje no se considera solo un vehículo para expresar las ideas, sino un factor que participa en la constitución de realidades sociales concretas (Santander, 2011). Para los investigadores del AD, el 
discurso tiene una gran capacidad de injerencia en las ideas y los hechos de las personas y comunidades. Esta visión del lenguaje nos permite entender lo discursivo como un modo de acción que tiene sus consecuencias, declaradas o no. En ese sentido, analizar el discurso mayoritario que circula en un determinado ámbito social (como es el caso de la escuela) es analizar una forma de intervención social. En definitiva, para que los modelos alternativos tengan más influencia en el cambio escolar, es necesario cuestionar el discurso pedagógico/ideológico de la escuela tradicional y sus consecuencias en las prácticas escolares, con el objetivo de que el profesorado desarrolle un conocimiento profesional crítico menos condicionado por la cultura dominante (Giroux, 1989; Howe y Stubbs, 1997).

\subsection{Definiendo el Discurso}

Sabemos que el lenguaje no es transparente, los signos no son inocentes, la connotación va con la denotación, que el lenguaje muestra, pero también distorsiona y oculta (Delord, 2015; Orlandi, 2012; Otha, 2015). Para poder tener en cuenta estas dimensiones nuestra analítica discursiva se ha basado en la teoría de M. Pêcheux (1995) y Orlandi (2012). Estos autores nos indican que el discurso nunca es exactamente lo que se está diciendo, pues dentro de él siempre existe: a) Una Formación Discursiva (FD), lo que literalmente se dice; b) Una Formación Ideológica (FI), la ideología en que se apoya lo que se dice y c) La Posición del Sujeto (PS), que explica como éste se posiciona dentro de esa ideología. Veamos un ejemplo con una afirmación ficticia: Yo no soy un maestro tradicional, a mí me gusta desarrollar las clases con explicaciones atractivas, con muchos colores, esquemas, a veces gasto unas bromas, para que los alumnos se queden con lo que yo estoy diciendo. Toda esta frase es una Formación Discursiva, dentro de ella encontramos la Formación Ideológica/Pedagógica tradicional, incluso aunque el profesor diga que yo no soy un maestro tradicional, pues su Posición como Sujeto está más cerca de un modelo tradicional que innovador: Para que los alumnos se queden con lo que yo estoy diciendo.

\subsection{Definiendo el discurso de la escuela tradicional}

El análisis del discurso de la escuela tradicional está descrito en los trabajos de Orlandi, 2012; Coracini, 2002 y Cauduro, 2011. Para Cauduro (2011), la escuela no es un contexto donde dialoguen diferentes discursos, ya que el profesor es el sujeto al que se le reconoce la posesión y el poder del conocimiento, él es el responsable de transmitirlo y el que juzga si los demás dicen lo que debe ser dicho y pensado. En el mismo sentido, Coracini (2002) afirma que el discurso tradicional impone dos funciones comunicativas que no coinciden con lo que se entiende por un dialogo discursivo:

- Obtener informaciones de fuentes únicas (el profesor o/y el libro texto)

- Obtener instrucciones para reproducir fielmente los mensajes del informante.

Es por esto que el discurso dominante de la escuela, y consecuentemente de la enseñanza de las ciencias, tiene una Formación Discursiva basada en el monólogo del docente, que posee la verdad absoluta y que se basa en materiales que deciden lo que debe ser aprendido. Además, el discurso tradicional posee una Formación Ideológica autoritaria, pues se basa en relaciones de poder jerárquicas y acríticas (Sead, 2013; Sennet, 2008).

\section{Definiendo el discurso innovador: El caso del P. IRES}

Para el análisis discursivo del P. IRES hemos tenido en cuenta los resultados de la tesis del Delord (2017), en la que fueron entrevistados los cuatro fundadores y analizados los materiales fundacionales del proyecto (Grupo Investigación en la Escuela 1991). En síntesis, el Modelo de Investigación en la Escuela (MIE), en el que se fundamenta el proyecto, considera el principio de 
investigación como elemento vertebrador del aprendizaje del alumnado y del desarrollo profesional del profesorado. El MIE pone a estudiantes y docentes en el centro del aprendizaje, escolar o profesional, y se organiza en torno a lo que se denomina Conocimiento Escolar y Conocimiento Práctico Profesional. El primero se considera un conocimiento epistemológicamente diferenciado del conocimiento cotidiano y del científico y trata de definir, a través de Itinerarios de Progresión, el conocimiento deseable para los estudiantes. El segundo es un conocimiento mediador entre las aportaciones de las disciplinas relacionadas con los procesos de enseñanza-aprendizaje y la acción docente. Después del estudio mencionado podemos afirmar que la Formación Discursiva del P. IRES pretende superar la pedagogía tradicional con un modelo fundamentado y con coherencia interna (Tabla 1). Su Formación Ideológica es liberadora y no sigue las normas del sistema educativo tradicional. Algunas de sus Posiciones Sujetos más significativas son: una visión problematizada y crítica de los contenidos, una metodología investigativa; una concepción de la evaluación no selectiva y sancionadora y una concepción de la enseñanza de las ciencias comprometida con el desarrollo social y la alfabetización científica.

TABLA 1. Diferencias entre el discurso tradicional y el del P. IRES

\begin{tabular}{|c|c|c|c|c|c|c|}
\hline ANÁLISIS & ¿Qué enseñar? & $\begin{array}{c}\text { ¿Cómo } \\
\text { enseñar? }\end{array}$ & $\begin{array}{l}\text { ¿Para qué } \\
\text { enseñar? }\end{array}$ & $\begin{array}{c}\text { ¿Cómo } \\
\text { evaluar? }\end{array}$ & \multicolumn{2}{|c|}{$\begin{array}{c}\text { CONCEPTOS } \\
\text { AD }\end{array}$} \\
\hline \multirow[t]{2}{*}{$\begin{array}{l}\text { Discurso } \\
\text { Tradicional }\end{array}$} & \multirow{2}{*}{$\begin{array}{l}\text { Contenidos } \\
\text { preestablecidos } \\
\text { concebidos } \\
\text { como verdades } \\
\text { acabadas, } \\
\text { fundamentalmen } \\
\text { te conceptuales }\end{array}$} & \multirow[t]{2}{*}{$\begin{array}{l}\text { Por } \\
\text { transmisión y } \\
\text { memorización } \\
\text { mecánica }\end{array}$} & \multirow{2}{*}{$\begin{array}{l}\text { Para el } \\
\text { siguiente } \\
\text { año y para el } \\
\text { mercado de } \\
\text { trabajo }\end{array}$} & \multirow[t]{2}{*}{$\begin{array}{l}\text { De forma } \\
\text { cuantitativa, } \\
\text { selectiva y } \\
\text { sancionadora }\end{array}$} & FD & Tradicional \\
\hline & & & & & FI & Autoritaria \\
\hline \multirow[t]{2}{*}{$\begin{array}{l}\text { Discurso P. } \\
\text { IRES }\end{array}$} & \multirow{2}{*}{$\begin{array}{l}\text { Contenidos } \\
\text { organizadores y } \\
\text { evolutivos. } \\
\text { Vinculados con } \\
\text { problemas } \\
\text { socio- } \\
\text { ambientales. } \\
\text { Incorporación } \\
\text { de } \\
\text { procedimientos } \\
\text { y valores. }\end{array}$} & \multirow[t]{2}{*}{$\begin{array}{l}\text { Basada en la } \\
\text { investigación } \\
\text { escolar }\end{array}$} & \multirow{2}{*}{$\begin{array}{l}\text { Para conocer } \\
\text { y resolver } \\
\text { críticamente } \\
\text { problemas } \\
\text { significativo } \\
\text { s de los } \\
\text { alumnos, de } \\
\text { la sociedad y } \\
\text { de la } \\
\text { naturaleza }\end{array}$} & \multirow[t]{2}{*}{$\begin{array}{l}\text { De forma } \\
\text { cualitativa, } \\
\text { formativa y } \\
\text { no } \\
\text { sancionadora }\end{array}$} & FD & Alternativa \\
\hline & & & & & FI & Libertadora \\
\hline
\end{tabular}

\section{Metodología}

Como hemos comentado, este trabajo es parte de una investigación más amplia sobre la génesis y evolución del P. IRES durante sus más de 30 años de existencia en el ámbito de la enseñanza de las ciencias, y la manera como sus miembros lo reinterpretan en la práctica docente. En este plan de investigación, uno de los objetivos ha sido conocer los obstáculos que dificultan la aplicación y extensión de los postulados alternativos del proyecto. Para ello escogimos a tres docentes que cumplieran los siguientes requisitos: trabajar en la misma ciudad (por razones logísticas), tener formación didáctica contrastada, larga experiencia docente y varios años de pertenencia al proyecto (personas asentadas en la profesión) y que cada uno fuera de un nivel educativo (Tabla 2), hicimos una serie de observaciones directa en sus aulas y una entrevista semiestructurada y en profundidad a cada uno para conocer las dificultades que plantea el cruce de una praxis alternativa en un contexto tradicional. En este artículo se presenta el análisis de las entrevistas en torno a los siguientes problemas: 
- ¿Qué obstáculos aparecen cuando se cruza el discurso tradicional con el discurso innovador de tres docentes del P. IRES en el ámbito de la enseñanza de las ciencias?

- ¿En qué aspectos el discurso innovador se mantiene en los docentes entrevistados?

- ¿Cómo superan o rodean los obstáculos identificados?

TABLA 2. Características de la muestra

\begin{tabular}{|c|c|c|c|c|}
\hline $\begin{array}{c}\text { Unidad } \\
\text { Didáctica }\end{array}$ & $\begin{array}{c}\text { Nivel y } \\
\text { Género }\end{array}$ & $\begin{array}{c}\text { Aplicación } \\
\text { del modelo } \\
\text { innovador } \\
\text { en clase }\end{array}$ & $\begin{array}{c}\text { Años en la } \\
\text { Red IRES/ } \\
\text { años de } \\
\text { experienci } \\
\text { a }\end{array}$ & $\begin{array}{c}\text { Características del } \\
\text { centro escolar }\end{array}$ \\
\hline Los tres cerditos & $\begin{array}{c}\text { Infantil, } \\
\text { maestra }\end{array}$ & Siempre & $9 / 14$ & $\begin{array}{c}\text { Escuela Pública. Centro } \\
\text { de la ciudad. Población de } \\
\text { clase media baja }\end{array}$ \\
\hline Universo & $\begin{array}{c}\text { Primaria, } \\
\text { maestro }\end{array}$ & Siempre & $6 / 15$ & $\begin{array}{c}\text { Escuela Pública. Barrio } \\
\text { humilde }\end{array}$ \\
\hline $\begin{array}{c}\text { Problemas socio- } \\
\text { ambientales e } \\
\text { historia del barrio }\end{array}$ & $\begin{array}{c}\text { Secundaria, } \\
\text { profesor de } \\
\text { secundaria }\end{array}$ & $\begin{array}{c}\text { Algunas } \\
\text { Veces }\end{array}$ & $11 / 18$ & $\begin{array}{c}\text { Escuela Pública. Zona } \\
\text { marginal. }\end{array}$ \\
\hline
\end{tabular}

Los sujetos fueron entrevistados en relación con las unidades didácticas de ciencias que estaban desarrollando: a) El cuento de los tres cerditos en E. Infantil (hábitat, alimentación, relación ser humano-naturaleza...); b) El universo en E. Primaria (de dónde venimos, la relación del universo con la naturaleza, la relación ser humano-universo...) y c) El estudio del barrio desde una perspectiva socio-ambiental en E. Secundaria (historia, evolución, condiciones del agua, basuras...) (Tabla 2).

El instrumento utilizado ha sido la entrevista semi-estructurada en profundidad (Gil, 2007) (Tabla 3). Para su diseño y aplicación tuvimos en cuenta los siguientes criterios (Díaz et al., 2013):

- Elaborar una guía de preguntas relacionadas con los problemas del estudio que permita activar el relato del entrevistado y promover que exprese el máximo de comentarios espontáneos.

- Elegir un lugar que favorezca la expresión del entrevistado, sin ruidos y otros estímulos que entorpezcan la entrevista.

- Explicar los propósitos de la entrevista y solicitar autorización para grabarla.

- Mantener una actitud respetuosa, no mostrando desaprobación ante los testimonios del entrevistado y tomando la palabra solo lo imprescindible para impulsar la narración.

- Con prudencia, invitar al entrevistado a explicar aspectos relevantes para el propósito del estudio.

Una vez transcritas las entrevistas realizamos el análisis utilizando los principios metodológicos del Análisis del Discurso y los presupuestos de una investigación cualitativa (Flick, 2009). Para ello se ha identificado en los tres textos la información relacionada con la Formación Discursiva, la Formación Ideológica y las Posiciones de los Sujetos. Cuando hemos observado que se cambiaban las PS, desviándose de su FD y su FI, hemos tratado de identificar los obstáculos que estaban actuando. En la Tabla 3 mostramos el guion de la entrevista. El orden en que están colocadas las preguntas no representa el orden en que fueron surgiendo en cada caso. 
TABLA 3. Preguntas del guion

\begin{tabular}{|l|}
\hline \multicolumn{1}{|c|}{ Preguntas } \\
\hline $\begin{array}{l}\text { ¿Cómo es el P. IRES?, ¿cuánto tiempo llevas implicado/a?, ¿cómo te } \\
\text { incorporaste y por qué?, ¿cuál es su relevancia para tu vida profesional? }\end{array}$ \\
\hline $\begin{array}{l}\text { ¿Cómo son tus clases?, ¿cómo planteas los contenidos?, ¿qué metodología } \\
\text { sigues? }\end{array}$ \\
\hline ¿Cómo influye el P. IRES en tus clases? \\
\hline ¿Con qué frecuencia y en qué momentos lo aplicas? \\
\hline Describe la unidad didáctica de ciencias que estas desarrollando \\
\hline ¿Cómo utilizas las ideas de los alumnos? \\
\hline ¿Cómo realizas la evaluación? \\
\hline ¿Qué facilidades encuentras al aplicar el P. IRES en tu escuela? \\
\hline ¿Qué dificultades encuentras al aplicar el P. IRES en tu escuela? \\
\hline $\begin{array}{l}\text { ¿Por qué utilizas la teoría y el Modelo de Investigación en la Escuela } \\
\text { (MIE) del P. IRES? }\end{array}$ \\
\hline
\end{tabular}

\section{RESULTADOS Y DISCUSIÓN}

Nuestro análisis se presenta de forma sintética en tres tablas, una por cada sujeto. En ellas presentamos los discursos de cada docente, distinguiendo la FD, la FI y las PS. Además, resaltamos las PS de los profesores que están condicionadas por el discurso tradicional, mostrando los obstáculos identificados, así como las PS que superan la FD y la FI del discurso tradicional.

\subsection{Análisis del sujeto 1}

El sujeto 1 (Tabla 4) es una maestra de E. Primaria que participa en el P. IRES desde hace 9 años y trabaja en una escuela pública situada en una zona de clase media baja de la ciudad de Sevilla. De la entrevista podemos inferir que tiene una FD innovadora y una FI liberadora, en la línea de los postulados del proyecto, incluso antes de incorporarse a él. Sin embargo, encontramos PS condicionadas por el discurso tradicional, por ejemplo, la necesidad de tener el apoyo de la dirección escolar para aplicar el proyecto: Yo trabajo de forma alternativa porque siempre he tenido la suerte de poder trabajar como yo quiero, en mi escuela la dirección me apoya y yo nunca he tenido problemas. Cuando analizamos la palabra suerte, podemos interpretar que si no tuviera ese apoyo tendría más dificultad para trabajar así. Aquí identificamos el obstáculo de dependencia jerárquica, pues la orientación del trabajo de la maestra está subordinada a la permisividad de la dirección. También observamos que está condiciona en sus Posiciones como Sujeto por el tipo de evaluación de la FD y la FI de la escuela tradicional, siendo éste un obstáculo identificado y no superado: Yo evalúo en un cuaderno con las letras apuntando regular, bueno, muy bien, convirtiendo sus notas en calificaciones sancionadoras (incluso no estando de acuerdo con ellas), condicionada por el marco normativo que prescribe la obligación de aprobar o suspender. 
TABLA 4. Análisis del Sujeto 1

\begin{tabular}{|c|c|}
\hline \multicolumn{2}{|r|}{ Sujeto 1} \\
\hline $\begin{array}{c}\text { FD } \\
\text { Alternativa/Innovadora }\end{array}$ & $\begin{array}{l}\text { Yo ya trabajaba de forma alternativa antes de conocer el } \\
\text { modelo IRES, pero después, a partir de este modelo, tuve más } \\
\text { seguridad y más organización metodológica. } \\
\text { Hace tiempo que trabajo de forma innovadora. } \\
\text { Descubrí el P. IRES y la Red por un amigo, pues hablaba } \\
\text { mucho con él de cómo cambiar la escuela. } \\
\text { Mis lecturas y las conferencias que buscaba hablaban sobre } \\
\text { la innovación, el cambio de la escuela [...] Tonucci era una } \\
\text { gran referencia para mí. }\end{array}$ \\
\hline FI Liberadora & $\begin{array}{l}\text { Mis alumnos con } 5 \text { años ya saben hacer mapas conceptuales, } \\
\text { saben investigar, argumentar, ser creativos. } \\
\text { Trabajo con el interés de los alumnos, ellos tienen ideas, } \\
\text { conocen mucho más de su alrededor do lo que imaginamos. } \\
\text { Ellos me dicen: podemos recoger esta información en la } \\
\text { biblioteca, podemos hacer tal cosa, son muy creativos. } \\
\text { Utilizo las ideas previas de los alumnos, pregunto lo que } \\
\text { quieren saber, yo apunto, después planificamos dónde vamos } \\
\text { a investigar y cómo vamos investigar. }\end{array}$ \\
\hline $\begin{array}{l}\text { PS condicionadas por el } \\
\text { discurso tradicional }\end{array}$ & $\begin{array}{l}\text { Yo trabajo de forma alternativa porque siempre he tenido la } \\
\text { suerte de poder trabajar como quiero, en mi escuela la } \\
\text { dirección me apoya y nunca he tenido problemas. } \\
\text { Evaluar a un niño es complicado porque cada uno tiene su } \\
\text { mundo, ellos están en formación, ¿quiénes somos nosotros } \\
\text { para limitar su imaginación?, pero procuro acercarme a cada } \\
\text { uno y ver cómo se comporta en cada tarea, con los números, } \\
\text { con las letras, etc. y lo evaluó en un cuaderno apuntando } \\
\text { regular, bueno, muy bien. } \\
\text { Es complicado poner un numerito a cada niño, yo lo pongo, al } \\
\text { final eso es importante para aprobar, pero intento siempre } \\
\text { hacer una calificación global e individual. }\end{array}$ \\
\hline Obstáculos encontrados & $\begin{array}{l}\text { 1. Dependencia jerárquica: la maestra asume que puede } \\
\text { trabajar de forma alternativa porque tiene suerte y recibe } \\
\text { apoyo directivo. A través del AD se interpreta que sin esa } \\
\text { suerte tendría más dificultades para trabajar así. } \\
\text { 2. Marco normativo: la maestra, incluso asumiendo que es } \\
\text { difícil evaluar a un niño, al final muestra una PS condicionada } \\
\text { por la FD y FI de la escuela tradicional y los evalúa de forma } \\
\text { sancionadora como regular, bueno y muy bien. }\end{array}$ \\
\hline Obstáculo superado & $\begin{array}{l}\text { Dependencia Jerárquica: al tener apoyo directivo la maestra } \\
\text { se siente protegida para aplicar el modelo. }\end{array}$ \\
\hline
\end{tabular}

\subsection{Análisis del sujeto 2}

El sujeto 2 (Tabla 5) forma parte de la dirección de una escuela pública de Educación Primaria de la ciudad de Sevilla en un barrio periférico. Él siempre trabaja con el P. IRES y lleva más de 6 años como miembro de la Red. Como se puede observar en la tabla 5, también este sujeto tiene una FD y una FI coherentes con las del proyecto. Sin embargo, cuando valora el supuesto de tener que cambiar de colegio a un centro privado afirma: Soy consciente de que si yo cambiara a 
una escuela privada tendría que trabajar a través del modelo ejército, todos iguales, pues así toca en la vida del profesor si no quiere perder su puesto de trabajo. Cuando habla de la evaluación dice: Sí, al final tengo que poner una nota, es que el sistema educativo te exige una nota individual y cuantitativa. En estas dos PS detectamos tres obstáculos: a) El de la dependencia jerárquica, según el cual el sujeto afirma que si trabajara en una escuela privada seguiría el modelo tradicional; b) El relativo a su condicionamiento laboral, por el que muestra la preocupación por su puesto de trabajo si mantuviera sus creencias en un contexto menos propicio y c) el producido por el marco normativo, según el cual hay que evaluar a los alumnos de forma cuantitativa; obstáculo éste último no superado.

TABLA 5. Análisis del Sujeto 2

\begin{tabular}{|c|c|}
\hline \multicolumn{2}{|r|}{ Sujeto 2} \\
\hline FD Alternativa/Innovadora & $\begin{array}{l}\text { Yo no consideraba las ideas de los alumnos, pero hacía } \\
\text { muchos experimentos. Ya no utilizaba el libro de texto, } \\
\text { pero el P. IRES me ayudó a tener un modelo didáctico. } \\
\text { Así que yo no estaba satisfecho con mis clases y } \\
\text { después del P. IRES empecé a utilizar un modelo en el } \\
\text { que creo y que es posible realizar en todas las clases. } \\
\text { Al principio parecía un monstruo destrozando los } \\
\text { contenidos para que los alumnos aprendieran mejor, no } \\
\text { tenía un método, no sabía lo que estaba haciendo, pero } \\
\text { sabía que quería cambiar, que el método tradicional no } \\
\text { estaba bien. } \\
\text { Me acuerdo que al principio pensaba que utilizaba las } \\
\text { ideas de los alumnos, porque al comienzo de un tema } \\
\text { les hacía preguntas y varios de ellos contestaban. Hoy } \\
\text { sé que utilizar las ideas de los alumnos es utilizarlas } \\
\text { durante todo el proceso. } \\
\text { Yo sabía que el profesor no debe transmitir los } \\
\text { contenidos. } \\
\text { Enseñaba sobre la luz a través del conocimiento } \\
\text { cotidiano de los alumnos [...] se enseña sobre la luz, } \\
\text { pero no a hacer la lectura de la factura de la luz de su } \\
\text { casa. }\end{array}$ \\
\hline FI Liberadora & $\begin{array}{l}\text { Siempre trabajo a través de las ideas de los alumnos, de } \\
\text { lo que es importante para él, para su vida. No trabajo } \\
\text { más con el libro texto, sino por proyectos que consisten } \\
\text { en contemplar la investigación a través de grandes } \\
\text { preguntas. } \\
\text { Primero pregunto las ideas iniciales, hacemos un } \\
\text { cuestionario, después hacemos una asamblea para ver } \\
\text { las ideas del grupo y después investigamos. } \\
\text { No me importa si sus ideas están equivocadas, él tiene } \\
\text { que saber defenderlas, para eso investigan. En relación } \\
\text { con las creencias religiosas, es complicado reconstruir } \\
\text { las ideas que traen de sus familias, por eso investigamos } \\
\text { lo que dicen los expertos. } \\
\text { Yo evalúo cómo las ideas de los alumnos van } \\
\text { evolucionando en el proceso. [...] es muy importante } \\
\text { saber argumentar en el aula. }\end{array}$ \\
\hline $\begin{array}{l}\text { PS condicionadas por el } \\
\text { discurso tradicional }\end{array}$ & $\begin{array}{l}\text { Bueno, soy consciente de que puedo trabajar de forma } \\
\text { alternativa en mi escuela, sin embargo, también soy } \\
\text { consciente de que si cambiara a una escuela privada }\end{array}$ \\
\hline
\end{tabular}




\begin{tabular}{|c|c|}
\hline & $\begin{array}{l}\text { tendría que trabajar a través del modelo ejército, todos } \\
\text { iguales, pues así toca en la vida del profesor si no } \\
\text { quiere perder su puesto de trabajo. } \\
\text { Intento evaluar al alumno de distintas formas, intento } \\
\text { hacer que él mismo haga su propia evaluación [...]. } \\
\text { Pero sí, al final tengo que poner una nota, es que el } \\
\text { sistema educativo te exige una nota individual y } \\
\text { cuantitativa. } \\
\text { Yo siempre explico a los alumnos lo que estoy } \\
\text { evaluando, es importante para ellos. También explico } \\
\text { para las familias cómo hacemos la evaluación [...] Si, } \\
\text { si, al final tengo que poner una nota. }\end{array}$ \\
\hline Obstáculos encontrados & $\begin{array}{l}\text { 1. Dependencia Jerárquica: En otro contexto asumiría } \\
\text { la jerarquía autoritaria, perdiendo autonomía } \\
\text { profesional. } \\
\text { 2. Condicionamiento laboral: Temer por la pérdida del } \\
\text { puesto de trabajo, adaptándose al modelo mayoritario. } \\
\text { 3. Marco normativo: Evaluar a través de la calificación } \\
\text { cualitativa para cumplir con la legislación vigente, } \\
\text { llevando a cabo prácticas contradictorias. }\end{array}$ \\
\hline Obstáculos superados & $\begin{array}{l}\text { Dependencia Jerárquica } \text { y Condicionamiento } \\
\text { laboral: Debido a que el profesor es del equipo } \\
\text { directivo se siente seguro para trabajar de modo } \\
\text { alternativo sin prejuicios profesionales. }\end{array}$ \\
\hline
\end{tabular}

\subsection{Análisis del sujeto 3}

El sujeto 3 (Tabla 6) trabaja en una escuela pública de E. Secundaria también en la ciudad de Sevilla, en un barrio marginal. Su escuela es la más humilde de los tres casos. El sujeto lleva más de 11 años en la Red IRES. También es este caso presenta una FD innovadora y una FI liberadora, coherentes ambas con el P. IRES. Sin embargo, no tiene apoyo directivo, así que las cuestiones jerárquicas y laborales son más evidentes que en los dos casos anteriores y son obstáculos que no están superados: Mi Director es un francotirador, él quiere, en realidad, que los maestros controlemos a los alumnos. El profesor afirma que no se pude ser un héroe y que en la lucha por cambiar la escuela el profesor innovador, que está casi siempre aislado, acaba perdiendo la batalla; al final no se puede exigir un acto heroico. De ahí que sus prácticas innovadoras y liberadoras las aplica: como yo puedo y cuándo puedo. Por último, aun estando en contra de la calificación, cambia su Posición y se amolda a la FD y FI del discurso tradicional: Bueno para mi es una barbaridad rotular a un alumno de bueno y malo con una nota [...], pero, al final, yo tengo que publicar las notas de forma cuantitativa por el programa Séneca.

TABLA 6. Análisis del Sujeto 3

\begin{tabular}{|c|l|}
\hline \multicolumn{1}{|c|}{ Sujeto 3} \\
\hline FD & $\begin{array}{l}\text { Yo antes de conocer el P. IRES sabía que mis clases no } \\
\text { Alternativa/Innovadoranaban, que mis alumnos repetían todo lo que yo decía y } \\
\text { eso no me hacía feliz. Hasta que conocí el proyecto, que tiene un } \\
\text { modelo que cuando te enteras y lo aplicas te das cuenta de que } \\
\text { es posible cambiar las clases. } \\
\text { Mis alumnos son de un barrio muy pobre, lo que yo intento } \\
\text { hacer es valorar el barrio y no marginalizarlos aún más. [...] } \\
\text { las personas de este barrio tienen que mentir y decir que no viven } \\
\text { aquí para conseguir trabajo. }\end{array}$ \\
\hline
\end{tabular}




\begin{tabular}{|c|c|}
\hline & $\begin{array}{l}\text { Una alumna me dice que ella no sirve para nada, eso es de los } \\
\text { otros profesores, que dicen eso a los alumnos que no les parecen } \\
\text { buenos. Así que siempre he intentado valorar a cada uno. [...]. } \\
\text { Tengo alumnos que trabajan, que están cansados, hay cuestiones } \\
\text { sociales muy duras dentro del aula que los profesores tienden a } \\
\text { no considerar. } \\
\text { Hay profesores que no conocen el barrio dónde trabajan, cogen } \\
\text { su coche, aparcan, hacen sus clases y vuelven a su realidad. }\end{array}$ \\
\hline FI Liberadora & $\begin{array}{l}\text { Yo intento hacer clases en que los alumnos aprendan sobre su } \\
\text { medio, su barrio, su historia, sus problemas ambientales, } \\
\text { incluso para valorarse ellos cómo personas, pues están en un } \\
\text { barrio muy marginado por la sociedad. Creo que eso es lo que } \\
\text { cómo maestro puedo hacer: que tengan orgullo de quienes son, } \\
\text { de donde vienen y que se den cuenta que la cuestión del barrio } \\
\text { también es una cuestión política; que al no estar este barrio a } \\
\text { los ojos de los turistas no recibe tanta ayuda como ocurre en el } \\
\text { centro. } \\
\text { Siempre acerco los contenidos a su realidad, trabajo con } \\
\text { cuestiones sociales y ambientales, [...] les hago reflexionar } \\
\text { sobre todos esto. } \\
\text { En un proyecto sacaron fotos del barrio y yo les enseñé que tras } \\
\text { ellas hay una historia y un contexto, y les pedi que lo } \\
\text { investigaran. }\end{array}$ \\
\hline $\begin{array}{l}\text { PS condicionadas por } \\
\text { el discurso tradicional }\end{array}$ & $\begin{array}{l}\text { Bueno, yo aplico el P. IRES siempre que me es posible, como yo } \\
\text { puedo y cuando puedo, porque mi Director es un francotirador, } \\
\text { él quiere que nos dediquemos básicamente a controlar a los } \\
\text { alumnos y a hacer muy bien las cosas burocráticas, rellenar los } \\
\text { papeles, poner las notas, etc. } \\
\text { El maestro innovador en realidad está solo en la escuela y en } \\
\text { esta lucha va a perder siempre. } \\
\text { El maestro está aislado contra todo el sistema, al final no se } \\
\text { puede pedir un acto heroico. } \\
\text { Bueno para mi es una barbaridad rotular a un alumno de bueno } \\
\text { y malo con una nota [...], pero, al final, yo tengo que publicar } \\
\text { las notas de forma cuantitativa por el programa Séneca. } \\
\text { La vida de un profesor de Secundaria es muy difícil. En realidad } \\
\text { lo que el sistema quiere es que estés con los alumnos } \\
\text { manteniendo el orden. Cuando vas hacer alguna cosa distinta te } \\
\text { ponen un montón de pegas y te hacen la vida muy difícil. Así que } \\
\text { casi es mejor no hacer nada. } \\
\text { Ellos quieren que tu rellenes papeles y estés presente en las } \\
\text { aulas. Es mentira cuando piden a los profesores que hagan } \\
\text { clases innovadoras. } \\
\text { La única escuela en que el P. IRES pude funcionar es en la } \\
\text { escuela x donde los directores son de la Red, en las demás es } \\
\text { muy dificil. }\end{array}$ \\
\hline $\begin{array}{c}\text { Obstáculos } \\
\text { encontrados }\end{array}$ & $\begin{array}{l}\text { 1. Dependencia Jerárquica: El profesor no puede trabajar } \\
\text { habitualmente conforme a su FD y FI pues no tiene apoyo del } \\
\text { director, lo que condiciona sus PS. } \\
\text { 2. Condicionamiento laboral: El profesor se siente aislado en la } \\
\text { escuela y admite que no pude tener una actitud heroica, tiene que } \\
\text { preservar su trabajo. } \\
\text { 3. Marco normativo: A pesar de que el sujeto no está de acuerdo } \\
\text { con la evaluación cuantitativa, la pone en práctica por una fuerte } \\
\text { limitación normativa. En este caso, observamos también que la }\end{array}$ \\
\hline
\end{tabular}




\begin{tabular}{|l|l|}
\hline & FD y FI de la escuela tradicional cambian la PS del profesor. \\
\hline Obstáculos superados & $\begin{array}{l}\text { Ninguno ha sido superado en este caso, básicamente por no tener } \\
\text { apoyo directivo y por la imposición legal. }\end{array}$ \\
\hline
\end{tabular}

Como podemos ver en estos casos, cuando dos discursos ideológicamente contrapuestos confrontan surgen obstáculos, en especial cuando uno es dominante, pues condiciona y limita al otro y a las Posiciones del Sujeto (acciones concretas y reales). También hemos visto que la muestra representa profesores con experiencia docente alternativa y con una concepción innovadora sólida. En bastantes de las unidades de información se observa cómo defienden y practican otro modelo de enseñanza y son conscientes de las limitaciones y los errores pedagógicos del modelo tradicional. No estamos, por tanto, ante sujetos que dudan o que están en transición, sus convicciones son claras, seguramente debido al soporte que les ha supuesto pertenecer al P. IRES. Sin embargo, en esta investigación nos interesa analizar, precisamente, los obstáculos que aparecen en sujetos con una posición bien definida desde un modelo alternativo al tradicional. Por eso, más allá de la actitud innovadora de estos docentes, debemos considerar los obstáculos aquí formulados: la dependencia jerárquica, el condicionamiento laboral y la limitación normativa en la evaluación, que están influyendo en que la innovación tenga menor capacidad de cambio.

Mención especial merece el obstáculo vinculado a las normativas sobre la evaluación, obstáculo no superado por los tres docentes, confirmando los resultados de otras investigaciones que identifican la evaluación como la dimensión más resistente al cambio (Olivos, 2011; Porlán et al., 2011; Rivero et al., 2017), al ser el elemento esencial que mantiene dos de las finalidades ocultas del sistema tradicional: a) el adiestramiento conductual de los estudiantes al estímulo externo de la calificación, que inhibe la expresión de ideas y modelos mentales propios y b) la selección social de aquellos estudiantes que, debido a sus ventajas de origen, a los apoyos familiares y al refuerzo de un papel escolar exitoso, son capaces de conseguir un aprendizaje significativo, más allá de que el modelo dominante promueva justamente lo contrario en la mayoría de sus compañeros.

\section{Conclusiones}

El objetivo de este artículo ha sido presentar los obstáculos que pueden estar impidiendo que una visión de la enseñanza de las ciencias coherente con las aportaciones de la investigación didáctica tenga mayor implantación en tres sujetos con un discurso innovador. Para ello nos hemos servido del Análisis de Discurso según M. Pêcheux (1995). A través de esta teoría sabemos que todo discurso posee una ideología subyacente. Además, que ideologías contrapuestas, cuando una de ellas es hegemónica, no consiguen establecer un diálogo, pues la dominante penetra en las Posiciones de los Sujetos generando obstáculos difíciles de superar. En el caso del sistema escolar, hemos considerado que la ideología innovadora/alternativa, que pretende la liberación y la autonomía de estudiantes y profesores, está también condicionada por la pedagogía tradicional.

Para poner a prueba este marco de referencia hemos analizado el discurso de tres docentes que tratan de llevar a la práctica en sus clases el Modelo de Investigación en la Escuela del P. IRES; proyecto con una sólida fundamentación y una larga tradición en el cambio de la enseñanza de las ciencias. Como resultado de este estudio, podemos dar respuesta a los problemas planteados:

¿Qué obstáculos aparecen cuando se cruza el discurso tradicional con el discurso innovador de tres docentes del P. IRES en el ámbito de la enseñanza de las ciencias?

a) La dependencia jerárquica, según la cual estos profesores están condicionados por la autoridad de la dirección escolar.

b) El condicionamiento laboral, por el que estos docentes temen poner en riesgo su estabilidad profesional. 
c) El marco normativo, según el cual las leyes, y sus prácticas administrativas, moldean las conductas de estos sujetos, especialmente en el caso de la evaluación.

¿En qué aspectos el discurso innovador se mantiene en los docentes entrevistados?

Hemos visto como los sujetos afirman que en las clases se debe trabajar a partir de un modelo investigativo centrado en el desarrollo de la autonomía de los estudiantes, tal como promueve el P. IRES. También reconocen el importante papel jugado por el proyecto para mantener y ampliar sus fortalezas en la innovación. Sin embargo, comprobamos cómo en los tres casos el discurso tradicional se impone al discurso innovador al abordar la evaluación, manifestándose ésta como el núcleo más resistente al cambio.

\section{¿Cómo superan o rodean los obstáculos identificados?}

Los tres sujetos afirman que pueden realizar o no sus innovaciones dependiendo del apoyo de la dirección y, por tanto, afirmamos nosotros, del grado de seguridad profesional y laboral que sienten al modificar las pautas convencionales. Dicho de otra manera, los docentes se atreven a cambiar cuando se sienten respaldados por la autoridad académica y, al contrario, viven con una sensación de riesgo (ser héroes) los intentos de cambio que van contra las directrices explícitas o implícitas de la misma.

Más allá de los problemas de investigación planteados, estos resultados nos llevan a algunas reflexiones más generales:

- Es importante que los docentes innovadores tengan un modelo de referencia y estén organizados en proyectos y redes de colaboración.

- La formación docente debe incorporar la reflexión social sobre el currículo y las prácticas educativas mayoritarias, especialmente en relación a cómo cambiar el modelo de evaluación de la escuela en los contextos tradicionales.

- Los profesores que desarrollan un modelo alternativo deben ser estimulados a participar en la administración escolar y los equipos directivos deben ser incentivados a apoyar a los profesores innovadores. En este sentido, la formación continua de los equipos directivos sigue siendo una línea estratégica para que los cambios tengan más repercusión.

- Sobre la evaluación es imprescindible contemplar alternativas legales basadas en la evaluación formativa, los informes cualitativos y el seguimiento de la evolución de las ideas de los alumnos, con el objetivo de retroalimentar y no sancionar el proceso de aprendizaje.

- Es necesario realizar más investigaciones acerca de esta problemática. En nuestro caso, estamos diseñando una futura investigación con otros proyectos innovadores de enseñanza de las ciencias y con muestras más amplias de cada nivel educativo, para ver sus dificultades al aplicarlos en el contexto tradicional.

\section{Referencias}

Althusser. L. (1989). A transformação da filosofia seguido de Marx e Lênin perante Hegel. São Paulo: Edições Mandacaru.

Álvarez-Uría, F. (2002). A escola e o espírito do capitalismo. En M.V. COSTA, (coord.). Escola básica na virada do século: Cultura, política e currículo. 3a. ed. São Paulo: Cortez.

Carbonell, J. (2002). A aventura de inovar: a mudança na escola. Porto Alegre: Artmed.

Cauduro, M.L.F. (2011). Escrita e Ensino: ecos do discurso pedagógico. São Carlos: Pedro \& João Editores.

Coracini, M.J. (2002). O jogo discursivo na aula de letras: língua materna e língua estrangeira. Campinas: Pontes.

Delord, G.C. (2015). Análise do Discurso com M. Pêcheux. En G.T.D. Guimarães, Ressignificando os labirintosda pesquisa qualitativa (pp. 95-110). Porto Alegre: Edipucrs, 
Delord, G.C. (2017). La innovación en la enseñanza de las ciencias. La identificación de obstáculos ideológicos: estudio de caso del Proyecto IRES (Tesis doctoral). Universidad de Sevilla.

Delord, G.C., Porlán, R. y Harres, J.B.S. (2017). La importancia de los proyectos y redes innovadoras para el avance de la enseñanza de las ciencias: el caso de un profesor de la Red IRES. Revista Eureka, 14(3), 653-665. DOI:10498/19514.

Delors, J. (2003). Educação: um tesouro a descobrir. Brasilia: Edigfies Asa.

Díaz, L., Torruco, U., Martínez, M. y Varela, M. (2013). La entrevista, recurso flexible y dinámico. Investigación en Educación Médica, 7(2), 162-167. Recuperado de http://www.redalyc.org/articulo.oa?id=349733228009.

Duit, R. y Treagust, D. (2003). Conceptual change: a powerful framework for improving science teaching and learning. International Journal of Science Education, 25(6), 671-688. DOI : $10.1080 / 09500690305016$

Ennis, R.H. (2011). The nature of critical thinking: An outline of critical thinking dispositions and abilities. Presentation at the Sixth International Conference on Thinking at MIT, Cambridge, MA, July, 1994. Recuperado de http://faculty.education.illinois.edu/rhennis/ documents/TheNatureofCriticalThink ing_51711_000.pdf.

Fiala, R. (2007). Educational Ideology and the School Curriculum. En A. Benavot, C. Braslavsky y N. Truong (eds.) School Knowledge in Comparative and Historical Perspective. Dordrecht: Springer.

Fisher, H.E., Borowski, A. y Tepner, O. (2012). Professional Knowledge of Science Education. En K. Fraser, B. Tobin y C. McRobbie (eds.), Second International Handbook of Science Education (pp. 771-782).

Flick, U. (2009). Introdução à pesquisa qualitativa. $3^{\mathrm{a}}$. ed. Porto Alegre: Artmed.

Fullan, M. (2001). The new meaning of education change. Nueva York: Teacher's College Press.

Garcia, P.S. (2010). Inovações, mudanças: Por que elas não acontecem na escola? São Paulo: LCT Editora.

Garrido, A. y Couso, D. (2013). Competència d'ús de proves científiques: Quines dimensions es promouen a les activitats de l'aula de ciències. Revista Ciències, 24, 42-47. Recuperado de https://ddd.uab.cat/record/106337 [10 de enero de 2018].

Gil, A.C. (2007). Como elaborar projetos e pesquisa. São Paulo: Atlas.

Giroux, H.A (1989). Los profesores como intelectuales. Barcelona: Paidós.

Grupo de Investigación en la Escuela (1991). Proyecto curricular IRES. Doc. I, II, III, IV. Sevilla: Díada.

Hamilton, D. (2002). O revivescimento da aprendizagem? Revista Educação e Sociedade, 78(2), 187-198.

Howe, A.C. y Stubbs, H.S. (1997). Empowering science teachers: a model for professional development. Journal of Science Teacher Education, 8(3), 167-182.

Harres, J.B, Wolffenbuttel, P.P. y Delord, G.C. (2013). Um estudo exploratório internacional sobre o distanciamento entre a escola e a universidade no ensino de ciências. Investigações em Ensino de Ciências (Online), 18, 365-365. Recuperado de https://www.if.ufrgs.br/cref/ojs/index.php/ienci/article/view/137/95. [22 de marzo de 2018].

Ibáñez, T. (2003). El giro lingüístico. En Análisis del Discurso. Manual para las ciencias sociales (pp. 21-42). Barcelona: Editorial UOC.

Lederman, N.G., Lederman, J.S. y Antink, A. (2013). Nature of science and scientic inquiry as contexts for the learning of science and achievement of scientic literacy. International Journal of Education in Mathematics, Science and Technology, 1(3), 138-147. Recuperado de http://files.eric.ed.gov/fulltext/ED543992.pdf. [10 de marzo de 2018].

Menezes, L. (2000). Comunicação na aula de Matemática e desenvolvimento profissional de professores. São Paulo: Millenium. 
Merchán, J. (2012). La introducción en España de la política educativa basada en la gestión empresarial de la escuela: el caso de Andalucía. Revista Archivos analíticos de políticas educativas, 20(1), 1-28. DOI: 10.14507/epaa.v20n32.2012.

Nogueira, R.C. (2009). La gubernamentalidad en los cursos del profesor Foucault. Revista Educação $\quad$ Realidade, 34(2), 21-34. Recuperado de http://seer.ufrgs.br/index.php/educacaoerealidade/article/view/8307/5539 [28 de marzo de 2018].

Olivos, T. (2011). La cultura de la evaluación y la mejora de la escuela. Perfiles educativos, 33(131), 116-130. Recuperado de http://www.scielo.org.mx/scielo.php?script=sci_arttext\&pid=S0185-26982011000100008 [10 de enero de 2018].

Orlandi, E.P. (2012). Discurso em análise: sujeito, ideologia e sentido. São Paulo: Pontes Editora.

Otha, T. (2015). O fim da miséria é só um começo? Noção de pobreza no discurso do plano Brasil Sem Miséria (Tesis doctoral). Pontifícia Universidade Católica do Rio Grande do Sul (PUCRS), Porto Alegre, Brasil.

Pêcheux, M. (1995). Semântica e discurso: uma crítica à afirmação do óbvio. Campinas: Unicamp.

Porlán, R. (1994). Constructivismo y Escuela. Sevilla: Díada.

Porlán, R., Martín Del Pozo, R., Rivero, A., Harres, J., Azcárate, P. y Pizzato, M. (2011). El cambio del profesorado de ciencias II: Resultados y conclusiones sobre la progresión de las concepciones didácticas. Enseñanza de las Ciencias, 29(3), 413-426.

Rivero, A., Martín Del Pozo, R., Solís, E., Azcárate, P. y Porlán, R. (2017). Cambio del conocimiento sobre la enseñanza de las ciencias de futuros maestros. Enseñanza de las Ciencias, 35(1), 29-52.

Santander, P. (2011). ¿Por qué y cómo hacer Análisis de Discurso? Revista Cinta Moebio, 41(1), 207-224. Recuperado de www.moebio.uchile.cl/41/santander.html [03 de marzo de 2018].

Sead, T. (2013). A invenção da psicologia social. Rio de Janeiro: Vozes.

Sennet, R. (2008). A cultura do novo capitalismo. São Paulo: Record.

Streck, D.R. (2009). Da pedagogia do oprimido às pedagogias da exclusão: um breve balanço crítico. Revista Educação \& Sociedade, 107(30), 539-560. DOI: 10.1590/S010173302009000200012.

Torres, J. (1991): El Curriculum oculto. Madrid: Morata.

Watts, M. y Jofili, Z. (1998). Towards critical constructivist teaching. International Journal of Science Education, 20(2), 173-185.

Wolffenbuttel, P.P., Harres. J.B y Delord, G.C. (2013). A formação de professores de física no programa pibid: análise da interação entre escola e universidade. Revista Contexto y Educação, 28(1), 106-133.

Cómo CitAR ESTE ARTí́culo

Delord, G. y Porlán, R. (2018). Del discurso tradicional al modelo innovador en enseñanza de las ciencias: obstáculos para el cambio. Didáctica de las ciencias experimentales y sociales, 35, 7790. DOI: 10.7203/DCES.35.12193 